\title{
Thermal log analysis for recognition of ground surface temperature change and water movements
}

\author{
M. Verdoya, P. Chiozzi, and V. Pasquale \\ Dipartimento per lo Studio del Territorio e delle Sue Risorse, Settore di Geofisica, Università di Genova, Viale Benedetto XV \\ 5,16132 Genoa, Italy
}

Received: 22 December 2006 - Published in Clim. Past Discuss.: 18 January 2007

Revised: 13 April 2007 - Accepted: 30 May 2007 - Published: 15 June 2007

\begin{abstract}
A joint analysis of surface air temperature series recorded at meteorological stations and temperature-depth profiles logged in nearby boreholes was performed to estimate conditions existing prior to the beginning of the instrumental record in central-northern Italy. The adopted method considers conductive and advective heat transport in a layered medium and provides simultaneous estimates of the preobservational temperatures and the Darcy velocities. The reconstruction of the ground surface temperature history using a generalised least-squares inversion method was performed for boreholes where hydrological disturbances to measured temperature logs were proved negligible. Both methods revealed generally coherent climatic changes in the whole investigated area. Climatic conditions were generally warm and comparable with the reference period 1960-1990. The absence of the Little Ice Age seems to be a generic feature of the climate in central-northern Italy. Climate change of the 19th century was generally insignificant with well-balanced periods of cold and warmth. The investigated area became significantly colder only at the end of the 19th century. Cooling culminated around 1950 when it was replaced by rapid warming. Recent warming was not inferred only for one of the investigated holes.
\end{abstract}

\section{Introduction}

The temperature variation at the Earth's surface can propagate, to some extent, downwards, sensibly affecting the underground temperature structure, which is mainly controlled by the terrestrial heat-flow density. Temperature-depth profiles measured within the uppermost hundred meters thus provide an archive of past climatic changes. Inversion of borehole geothermal data can reveal the ground surface tem-

Correspondence to: M. Verdoya

(verdoya@dipteris.unige.it) perature (GST) history (see e.g. Beltrami and Harris, 2001; Pasquale et al., 2005a, and references therein) which for deeper boreholes can be reconstructed back to the last ice age (e.g. Kukkonen and Joeleth, 2003). Another method to unravel the climate changes from geothermal data is based on the joint processing of surface air temperature (SAT) recorded at meteorological observatories and borehole temperature profiles to obtain the pre-observational mean temperature (POM), against which the recent climate changes can be referenced (Chisholm and Chapman, 1992; Harris and Chapman, 1998; Pasquale et al., 1998). The POM method is based on the hypothesis that, on the long temporal scale, the mean annual GST tracks the mean annual SAT.

The foregoing past climate reconstruction techniques have been based on the assumption of negligible hydrological activity at the investigated boreholes, i.e. a pure conductive heat transfer. In practice, screening of the geothermal data is performed before processing, and only undisturbed data or thermal logs corrected for the hydrological disturbances are used for climatic reconstructions. Because of the general lack of satisfactory hydrological information in the majority of the borehole sites, the screening procedure is not an easy task. Recent investigations recognised the strong correlation between SAT and GST variations, but also stressed the role of groundwater flow, which may alter or mimic the climatically produced signal (Ferguson et al., 2003; Reiter, 2005; Majorowicz and Safanda, 2005; Verdoya et al., 2007). Moreover, it was argued that some of the previous GST reconstructions were carried out without sufficient justification of the absence of groundwater flow.

Figure 1 illustrates with a theoretical example how vertical flow in a permeable rock may disturb the conductive geotherm. Hydraulic disturbances may produce a distortion of geotherms similar to that caused by a climatic change (see e.g. Bodri and Čermák 2005 for details). Recharge (downward groundwater movement) results in concave upward temperature-depth profiles, while discharge (upward

Published by Copernicus Publications on behalf of the European Geosciences Union. 

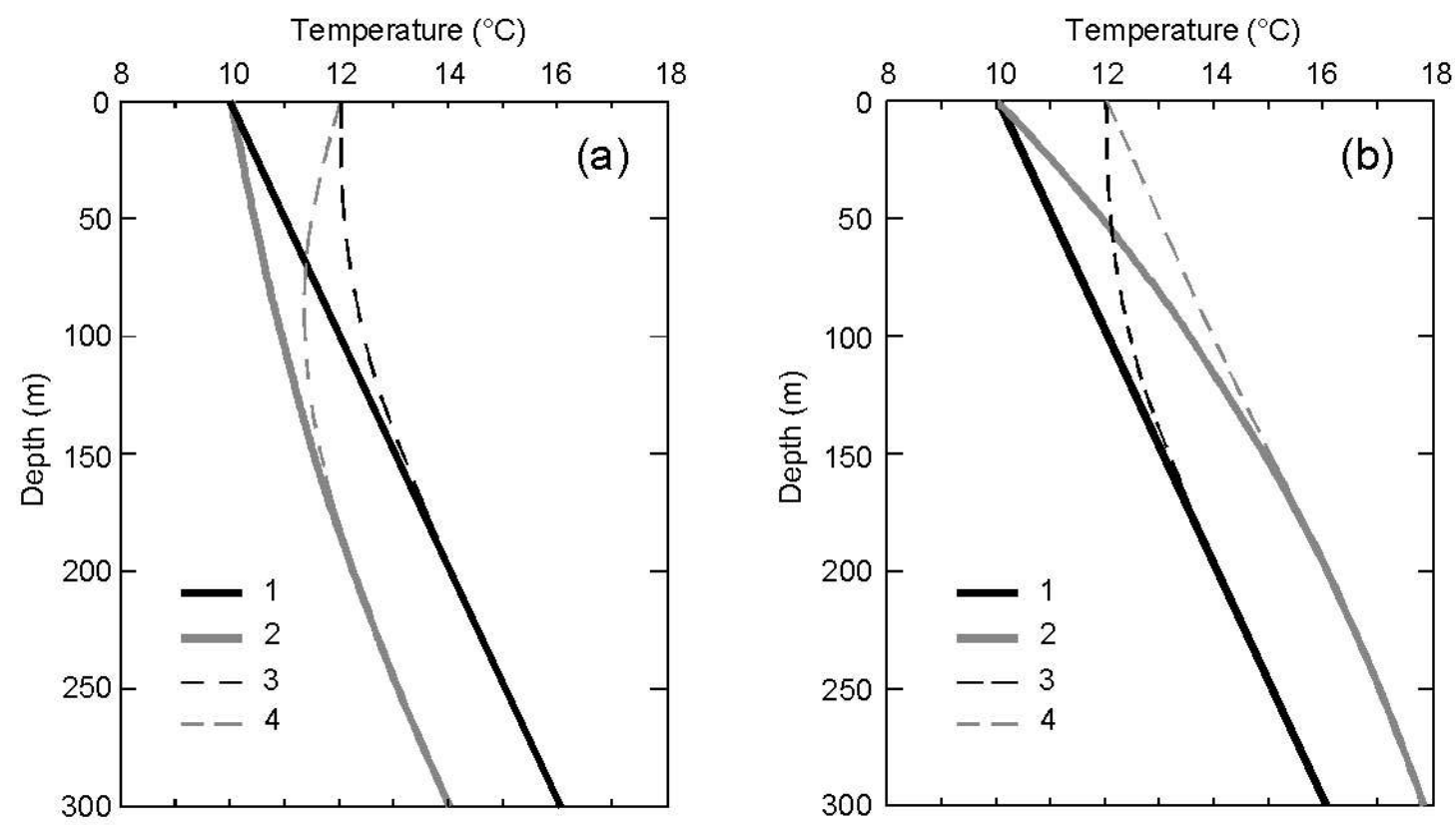

Fig. 1. Models of temperature-depth profiles illustrating deviations from a pure conductive geotherm caused by a GST change combined with downward (a) and upward (b) groundwater flow. Thermal regimes: 1) conductive, 2) advective, 3) conductive climatically perturbed, 4) advective plus climatic effect. Model parameters: thermal diffusivity $31.5 \mathrm{~m}^{2} \mathrm{yr}^{-1}$, thermal conductivity $2.5 \mathrm{~W} \mathrm{~m}^{-1} \mathrm{~K}^{-1}$, conductive temperature gradient $20 \mathrm{mK} \mathrm{m}^{-1}$, Darcy velocity $2.0 \times 10^{-9} \mathrm{~m} \mathrm{~s}^{-1}$ (negative upward), porosity $1 \%$. The climate perturbation is produced by a $2 \mathrm{~K}$ increase of GST occurred 100 years ago.

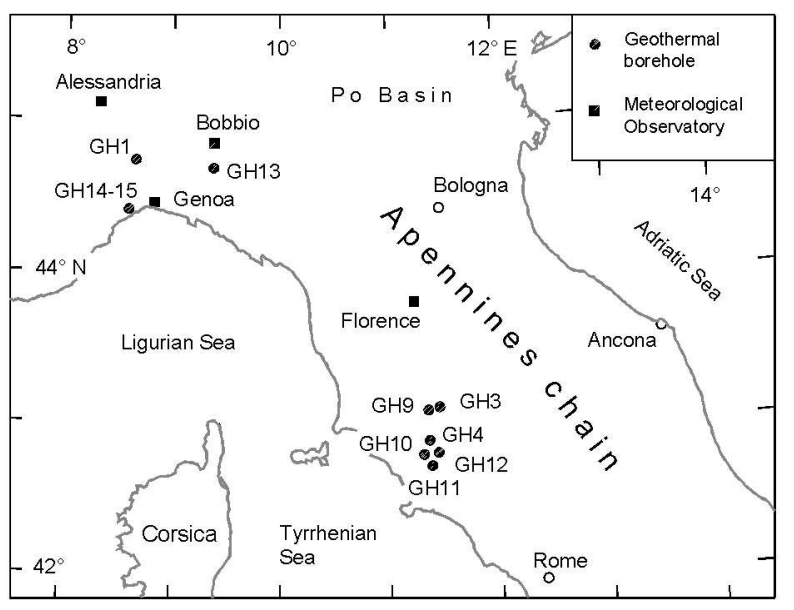

Fig. 2. Location of borehole sites and meteorological stations in central-northern Italy.

flow) creates an opposite effect. Relatively high Darcy velocities $\left(10^{-8} \mathrm{~m} \mathrm{~s}^{-1}\right)$ emphasize the effect of water flow. However, Lewis and Wang (1992), investigating a set of boreholes located in a relatively small area (Lac Dufault, Canada), showed that even small water flows can produce large uncertainty in the inference of the onset of recent warming.

Bodri and Čermák (2005) proposed a new method, which incorporates advective disturbances due to vertical ground water flow in the usual conductive approach for POM estimates. A series of tests both on synthetic and field examples indicated that inclusion of advective heat transfer in the inference of the POM temperature can provide more reliable estimates, thus making possible to use for past temperature reconstructions a vast amount of previously rejected temperature logs. Since no direct hydrogeological information is often available for a geothermal borehole, the foregoing method can also be used for testing the magnitude of groundwater flow.

In this work we apply such an advective/conductive approach for the past climate reconstruction to a set of temperature-depth profiles of central-northern Italy (Fig. 2). Some of these profiles were rejected in previous studies on climate change (Pasquale et al., 2000, 2005a), because more or less affected by perturbations due to groundwater. The identification of the past climate changes in this area by using only meteorological data is problematic because of the relative shortness of the time series, the sparse spatial arrangement of the existing SAT records and the possible presence of hidden heterogeneities in the SAT series. Data from nearby boreholes not affected by groundwater disturbances were used for the verification of the obtained results. 
Table 1. List of the boreholes located in Fig. 2 together with time of the temperature measurements.

\begin{tabular}{llllllll}
\hline Site & Well & Year & Lat. N & Long. E & Alt. (m) & Depth (m) & Ref. $^{\text {a }}$ \\
\hline Acqui Terme & GH1 & 1996 & $44^{\circ} 40.6^{\prime}$ & $08^{\circ} 27.2^{\prime}$ & 152 & 200 & 1 \\
Fontalcinaldo & GH3 & 2000 & $43^{\circ} 07.0^{\prime}$ & $11^{\circ} 29.4^{\prime}$ & 720 & 358 & 1 \\
Murci & GH4 & 1999 & $42^{\circ} 45.3^{\prime}$ & $11^{\circ} 24.3^{\prime}$ & 380 & 200 & 1 \\
Castiglione del Bosco & GH9 & 1993 & $43^{\circ} 05.9^{\prime}$ & $11^{\circ} 26.2^{\prime}$ & 175 & 150 & 2 \\
Podere Castagnolo & GH10 & 1992 & $42^{\circ} 43.5^{\prime}$ & $11^{\circ} 24.2^{\prime}$ & 190 & 152 & 2 \\
Fattoria Pomonte & GH11 & 1991 & $42^{\circ} 38.6^{\prime}$ & $11^{\circ} 25.8^{\prime}$ & 170 & 155 & 2 \\
Usi & GH12 & 1995 & $42^{\circ} 43.6^{\prime}$ & $11^{\circ} 27.6^{\prime}$ & 330 & 147 & 2 \\
Marsaglia & GH13 & 1982 and 2002 & $44^{\circ} 43.0^{\prime}$ & $09^{\circ} 23.0^{\prime}$ & 305 & 146 & 3 \\
Lerca 1 & GH14 & 2001 and 2002 & $44^{\circ} 24.2^{\prime}$ & $08^{\circ} 37.8^{\prime}$ & 130 & 60 & 3 \\
Lerca 2 & GH15 & 2001 and 2002 & $44^{\circ} 24.7^{\prime}$ & $08^{\circ} 38.0^{\prime}$ & 105 & 70 & 3 \\
\hline
\end{tabular}

a 1, Pasquale et al. (2005a); 2, Baldi et al. (1994); 3, this paper.

\section{Thermal records}

The selected dataset consists of seven temperature-depth profiles recorded in boreholes located in the LigurianTyrrhenian side of the Apennines chain, Italy, four (GH9GH12) in the central sector and three (GH13-GH15) in the northern part (Fig. 2). The boreholes were drilled both for studies of surface heat-flow density and exploration of geothermal resources and water. They are situated in the vicinity of some boreholes (GH1, GH3 and GH4) unaffected by advective perturbations, which were analysed in previous papers (Pasquale et al., 2000, 2005a; Bodri et al., 2003). Table 1 shows geographic coordinates and date of logging of the boreholes. The temperature-depth distributions of the seven boreholes are shown in Fig. 3.

\subsection{Central sector}

The boreholes GH9-GH12 were drilled in a region of remarkable interest for geothermal energy, and characterized, on the regional scale, by high terrestrial heat-flow density values $\left(>100 \mathrm{~mW} \mathrm{~m}^{-2}\right)$. GH10-GH12 are very close one to each other and situated within a few kilometres from the well-known Larderello geothermal field. The hole GH9 is about $35 \mathrm{~km}$ far from the three holes. The available temperature-depth profiles, together with those previously analysed from boreholes GH3-GH4, form a robust dataset to characterize climatic changes in a relatively wide area of the central Tyrrhenian side.

Temperatures were measured to a maximum depth of $155 \mathrm{~m}$ depth with a sampling interval of $10 \mathrm{~m}$ (Baldi et al., 1994). The holes GH9-GH11 penetrated clayey strata, while the stratigraphy in the borehole GH12 consists of shales, limestones and marly limestones. High values of heat-flow density $\left(80-160 \mathrm{~mW} \mathrm{~m}^{-2}\right)$ were inferred in these boreholes.

No hydrogeological information is available for the area surrounding the investigated holes. Thus, the preliminary considerations about the possible presence of groundwater
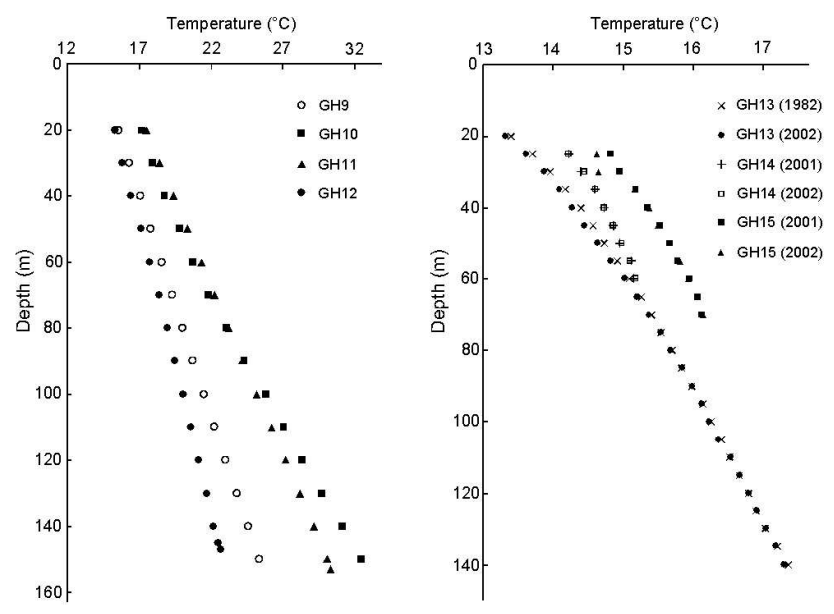

Fig. 3. Temperature-depth distributions of the boreholes of Fig. 2.

flow can only be done on the base of indirect information. The holes are located in areas with small elevation variations $(<200 \mathrm{~m})$ within a radius of few kilometres. Water flow through pores and/or small fractures might be driven either by hydraulic head differences or free convection resulting from the 10-20 K temperature differences between the hole top and bottom (Fig. 3) that can produce variations in the fluid density of $2.0-4.5 \mathrm{~kg} \mathrm{~m}^{-3}$. Geothermal observations and model calculations showed that such a water flow is generally very slow, especially in clayey rocks, which are characterised by low hydraulic conductivity (see e.g. Bodri and Dövényi, 2004, and references therein).

The temperature perturbations in the measured profiles caused by climate change were estimated from the analysis of the reduced temperatures, defined as the departure of the observed temperature-depth data from the background thermal regime. The latter is inferred from the deeper part $(>100-150 \mathrm{~m})$ of temperature logs, which is, in practice, more or less linear and considered as unaffected by climatic 
Table 2. Pre-observational mean temperatures before the year 1814 for pure conductive approach ( $\mathrm{POM}_{C}$ ) and for conductive and advective approach $\left(\mathrm{POM}_{a}\right)$, and the corresponding Darcy velocity $v$ (negative value corresponds to upward flow). Uncertainty on POMs is $<0.1^{\circ} \mathrm{C}$.

\begin{tabular}{llllll}
\hline Borehole & $T_{o}\left({ }^{\circ} \mathrm{C}\right)$ & $G_{o}\left(\mathrm{mK} \mathrm{m}^{-1}\right)$ & $\operatorname{POM}_{c}\left({ }^{\circ} \mathrm{C}\right)$ & $\operatorname{POM}_{a}\left({ }^{\circ} \mathrm{C}\right)$ & $\mathrm{v}\left(10^{-9} \mathrm{~m} \mathrm{~s}^{-1}\right)$ \\
\hline GH9 & 13.7 & 77.3 & 15.0 & 15.1 & -0.03 \\
GH10 & 12.3 & 134.5 & 15.2 & 14.7 & 0.28 \\
GH11 & 15.4 & 97.7 & 15.0 & 15.0 & 0.01 \\
GH12 & 15.2 & 49.2 & 16.0 & 16.8 & -0.79 \\
\hline
\end{tabular}

$T_{o}$, temperature at zero depth, $G_{o}$, temperature gradient.
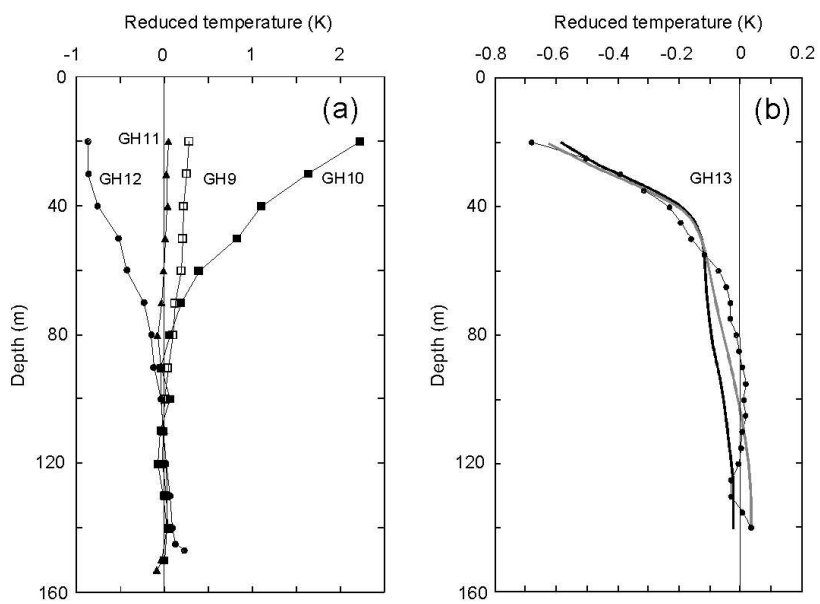

Fig. 4. Reduced temperatures for boreholes GH9-GH12 (a) and GH13 (b). The best-fit calculated temperatures are shown for GH13: black curve, pure conductive; grey curve, conductive/advective approach.

perturbations (e.g. Chisholm and Chapman, 1992). Thus, the departure decreases with depth. Figure 4a shows reduced temperatures for the holes GH9-GH12 while Table 2 displays the parameters of the background temperature field, calculated with the standard linear regression technique.

Apparently, three boreholes denote recent warming, which seems clearer in the borehole GH10, whereas the reduced temperature of GH12 shows cooling. Since climatic changes from different sites in a relatively small area are expected to be coherent, the presence of hydrogeological disturbances is highly probable.

\subsection{Northern sector}

Borehole GH13 was drilled for geothermal studies within homogenous claystone strata. Temperature was measured to $143 \mathrm{~m}$ depth with a $5 \mathrm{~m}$ reading interval. A precision temperature acquisition system was used with a 4-wire shielded cable and a Pt-resistance sensor with a total system uncertainty of $0.01-0.03^{\circ} \mathrm{C}$. Since the first temperature logging in 1982 , the hole was periodically monitored, and the last tempera- ture profile was carried out in 2002 (Fig. 3). No appreciable temperature variation during this relatively long period has been observed, and the data indicate recent cooling, which appears even more evident in the reduced temperature plot (Fig. 4b). Also the parameters extrapolated from the lowermost part of the measured temperature logs are almost coinciding $\left(T_{o}=13.5\right.$ and $13.6^{\circ} \mathrm{C}, G_{o}=27.1$ and $26.8 \mathrm{mK} \mathrm{m}^{-1}$ for the 1982 and $2002 \operatorname{logs}$, respectively).

Boreholes GH14 and GH15 were logged both in April 2001 and January 2002 (Fig. 3). Temperatures were measured with the same equipment used for GH13, down to 60 and $70 \mathrm{~m}$ depth, respectively, with reading interval of $5 \mathrm{~m}$. GH14 crossed mainly marls with a thin narrow layer of alternating marls and sandstones from 33 to $38 \mathrm{~m}$ depth. GH15 penetrated mostly conglomerates, with an intercalated thin layer of marl and sandstone between $28-30 \mathrm{~m}$, and serpentines at $69 \mathrm{~m}$ depth. Both pairs of temperature logs coincide well, except for the uppermost part of the GH15 log, where temperatures recorded in January 2002 are lower, probably due to meteoric water infiltration within the fractured conglomerate. Since both boreholes are very shallow, in principle only the most recent climatic signal can be recovered. Moreover, the steady-state surface temperature $T_{o}$ and the unperturbed temperature gradient $G_{o}$ cannot be extrapolated from the lower part of the measured temperature logs, but they must be estimated together with the other unknown parameters, namely the POM-value and the Darcy velocity.

\section{SAT data}

Four time series of annual surface air temperature (SAT) were used as forcing functions for estimating POMs as well as for the verification of the reconstructed GST histories (Fig. 5). The longest records are available for the stations located in Genoa (1833-2002) and Florence (1814-1994). Shorter series are available for Alessandria (1854-1964) and Bobbio (1934-2002). Details on data homogenisation, updating and improvement are scattered in several papers (see Pasquale et al., 2005a, and references therein). We performed additional tests by means of diagrams of year-by-year temperature variability, defined as the absolute difference of the average temperature from one year to the next (Bodri and 

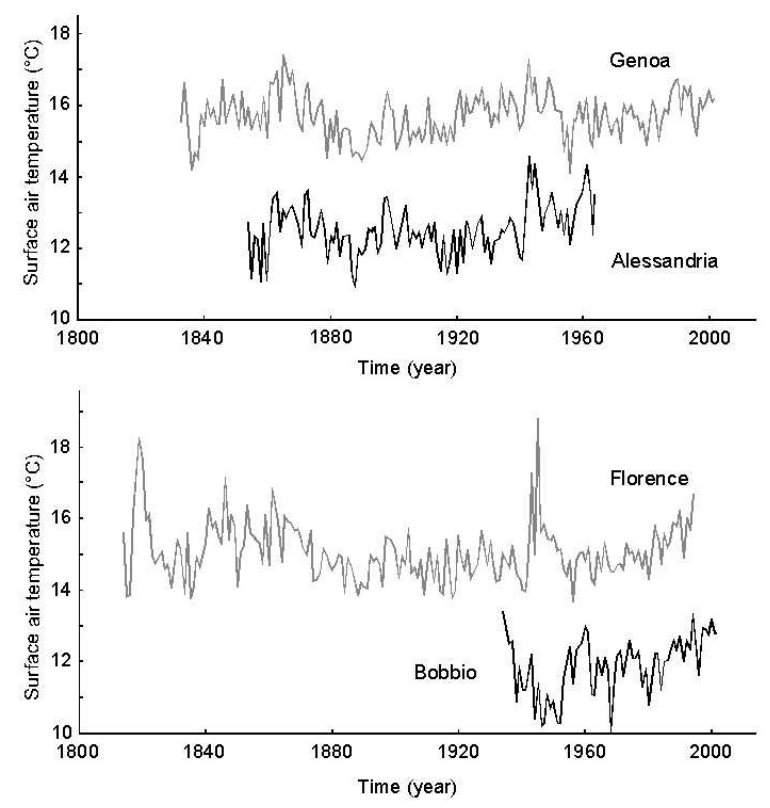

Fig. 5. Mean annual SAT at meteorological stations of Florence, Genoa, Alessandria and Bobbio.

Čermák, 2003). At all meteorological stations temperature oscillations are highly variable, warming and cooling trends often alternating. Moreover, our analysis shows a slight decrease in temperature variability, which is common in many SAT series of the Northern Hemisphere (Karl et al., 1995; Moberg et al., 2000; Bodri and Čermák, 2003). Figure 6 shows the interannual variation and trend of the average temperature for the Florence and Genoa time series.

Generally, temperature measurements at a given meteorological station are representative of an extensive area. Thus, POMs can be estimated from SAT data recorded at observatories that need not to be so close to the investigated boreholes. However, in the Italian peninsula, the barrier effect of the $\mathrm{N}-\mathrm{S}$ trending Apennines ridge and the destabilising effect of the Mediterranean Sea are the two main geographical factors that imply strong effects of the local air circulation, resulting in high spatial climate variability (Pasquale et al., 2005b).

According to Hansen and Lebedeff (1987), for station pairs with at least 50 years of common records, selected randomly among stations at latitudes between $23.6^{\circ} \mathrm{N}$ and $44.4^{\circ} \mathrm{N}$, and from $44.4^{\circ} \mathrm{N}$ to $64.2^{\circ} \mathrm{N}$, the correlation coefficient of the annual mean temperature variation is larger than 0.5 if distances for each direction, defined by $45^{\circ}$ intervals, are less than 750 and $1250 \mathrm{~km}$, respectively. At middle and high latitudes the correlation approaches unity as the stations separation becomes small. In Italy, the area size for which a given station data may provide significant information on temperature change is smaller and substantially depends on direction. The correlation between SAT series from Florence and Genoa (about $200 \mathrm{~km}$ apart) is 0.70. Alessandria, Bob-
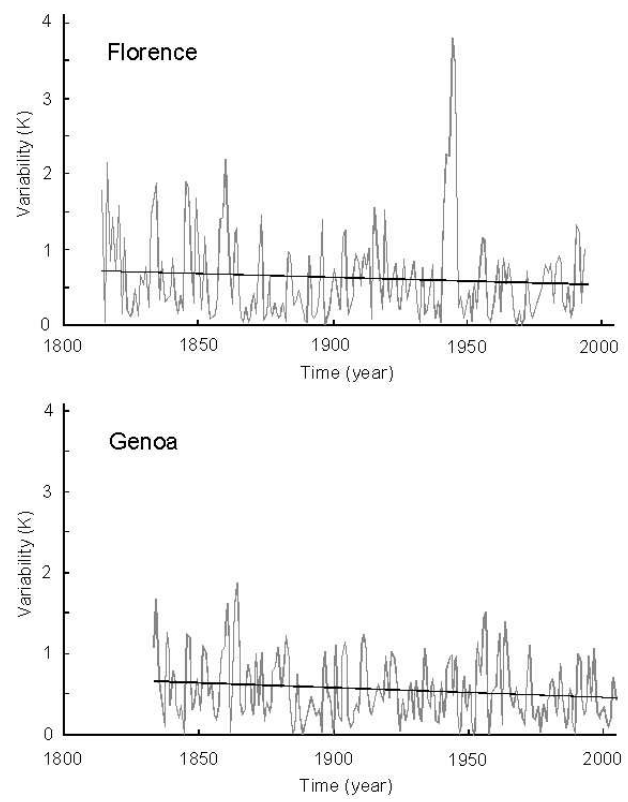

Fig. 6. Interannual temperature variability and trend for Florence and Genoa SAT time series. Differences in absolute value.

bio and Genoa observatories are arranged as a triangle with $50-60 \mathrm{~km}$ long sides. Nevertheless, due to the position of the stations with respect to the Apennines watershed, the correlation of Genoa and Alessandria SAT series is 0.66 and only 0.21 for Genoa and Bobbio. This fact demonstrates the need of a careful choice for the SAT series to be used as forcing functions in POM calculations.

\section{Climate change reconstructions}

We used the approach described by Bodri and Čermák (2005) for the joint estimation of the POM temperature and the vertical velocity of fluid migration. The thermal effect of water flow was analysed in the 1-D case by taking into account both conductive and advective heat transfer in a homogeneous semi-infinite medium. For a uniform groundwater flow, the variation of temperature $T$ with time $t$ and depth $z$ (positive downward) is

$k \frac{\partial^{2} T}{\partial z^{2}}-\rho_{w} c_{w} v_{z} \frac{\partial T}{\partial z}=\rho c \frac{\partial T}{\partial t}$

where $c_{w}$ and $\rho_{w}$ are the specific heat and density of water, respectively, $c, \rho$ and $k$ are the specific heat, density and thermal conductivity of the rock-water complex, respectively, and $v_{z}$ is the Darcy velocity in the vertical direction.

For convenience, this equation can be treated in terms of reduced temperature, and its solution is a combination of the conductive (climatic) and advective heat transport. The temperature perturbation for a surface temperature history composed of $n$ individual step changes of amplitude $\vartheta_{i}$ and time 


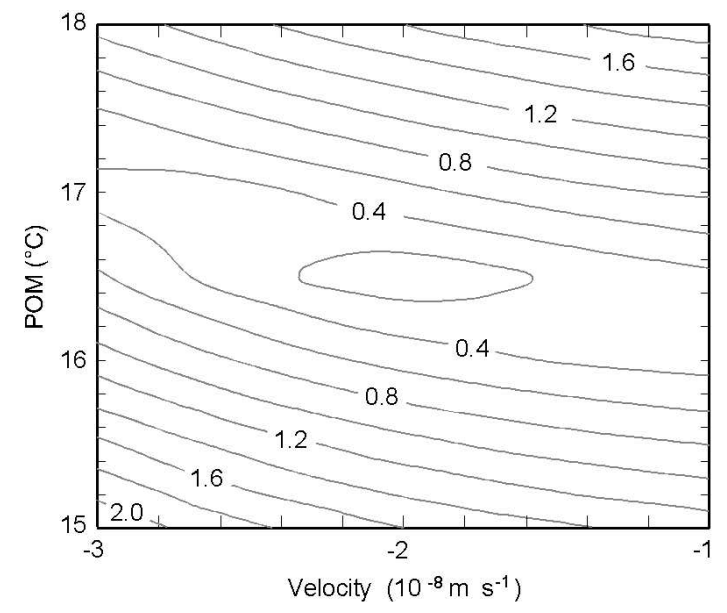

Fig. 7. Map of the rms misfit (in ${ }^{\circ} \mathrm{C}$ ) for a possible range of POM and Darcy velocity for the borehole GH14. The best fitting values correspond to the rms minimum.

$t_{i}$ prior to the measurement of the thermal profile is (Carslaw and Jaeger, 1959)

$$
\Delta T_{c}(z)=\sum_{i=1}^{n} \vartheta_{i} \operatorname{erfc}\left(\frac{z}{\sqrt{4 \chi t_{i}}}\right)
$$

where "erfc" is the complementary error function and $\chi$ is the thermal diffusivity.

For boundary conditions $T=T_{o}$ at depth $z=0$ and $T=T_{L}$ at $z=L$, the temperature perturbation due to advection is (Bredehoeft and Papadolpulos, 1965)

$\Delta T_{a}(z)=\left(T_{L}-T_{o}\right)\left(\frac{\exp (\beta z / L)-1}{\exp (\beta)-1}\right)-G_{o} z$

where $\beta=\left(\rho_{w} c_{w} v_{z} L\right) / k$ and $G_{o}$ is the background temperature gradient.

The unknown parameters, POM temperature and fluid vertical velocity, can be obtained by comparing the observed reduced temperatures with synthetic temperature logs calculated using SAT record as an upper boundary condition. We used the steepest descent algorithm minimizing the sum of squared differences between measured and calculated reduced temperatures. The most reliable values of POM and groundwater velocity are those corresponding to the minimum root mean square (rms) misfit. Figure 7 shows as an example the rms misfit map for the borehole GH14.

The adopted method can be applied only if permeability does not exceed a critical value of $1-5 \times 10^{-15} \mathrm{~m}^{2}$. At higher permeability, upward flow would cause surface warming-up, thus violating the chosen upper boundary condition. The flow velocity corresponding to the critical permeability can be easily estimated since it is linearly scaled to surface hydraulic head. At hydraulic heads characteristic for the investigated area, the maximum Darcy velocities that could provide reliable POM values amount to $1-5 \times 10^{-9} \mathrm{~m} \mathrm{~s}^{-1}$.
For the boreholes where advective disturbances resulted of minor or negligible importance, we also reconstructed the GST history by means of the generalised least-square inversion method described by Bodri and Čermák (1995), which is based on a 1-D purely conductive thermal regime in a heatsource free, layered medium. This method does not impose any explicit constraints on the GST history and assumes thermal properties and basal heat-flow density to be known. The surface temperature history is approximated by a series of unequal time intervals of constant temperatures, which represent unknowns of the problem. The surface temperature history is approximated by a series of time intervals where temperatures are assumed to be constant. The width of these intervals usually increases going back in time. However, the appropriateness of this parametrization is checked with the help of the resolution matrix (e.g. Menke, 1989) calculated a posteriori. This allows the construction of a GSTH parametrization compensating for the decrease in resolution with time.

Even a relatively complicated and long GST history can be approximated by a limited number of parameters, without losing details of the recent climate change. A series of tests carried out on synthetic temperature-depth data show that the reliability of the reconstructed GST history is about 10$15 \%$ for a change occurred 50 years ago, whereas changes occurred 200-300 years ago can be resolved with the same reliability only for 50 years intervals (Bodri and Dövényi, 2004).

\subsection{POM temperatures and Darcy velocity}

The POM analysis for boreholes GH9-GH12 was made by using the mean annual temperatures measured at the Florence meteorological station as representative SAT record. This time series shows warm conditions from 1814 to 1870 , a cold period from 1880s to 1940 s, and very high temperatures between 1940 and 1950, followed by a short period (1953-1957) with the lowest temperatures. Since then, gradual warming has been occurring at a rate of $0.04 \mathrm{~K} \mathrm{yr}^{-1}$. The mean temperature in the 1960-1990 reference period is $15.0^{\circ} \mathrm{C}$.

Results of POM estimations for the four boreholes of the central sector of Italy are summarized in Table 2. Results show that the effect of water movement is almost negligible in boreholes GH9 and GH11. Noticeable water flow was inferred in GH10 and GH12. The pre-observational mean temperature estimated using a pure conductive approach $\left(\mathrm{POM}_{c}\right)$ is $0.5 \mathrm{~K}$ higher than that obtained with a conductive/advective model $\left(\mathrm{POM}_{a}\right)$. In the boreholes GH9 and GH11, the $\mathrm{POM}_{a}$ values agree well with those obtained assuming purely conductive heat transfer. The boreholes GH9GH11 give mean temperatures prior to 1814, which are comparable with the average temperature of the reference period, i.e. long-term pre-observational conditions similar to the 1960-1990 decades. 
Table 3 shows the POM temperatures obtained considering SAT data only since 1900 . These results, representing the long-term pre-observational conditions before the beginning of the 20th century, are in substantial agreement with those obtained using the entire SAT time series, thus indicating that climatic conditions have been relatively constant throughout the 19th century. In other words, the 19th century does not contain definite trends, with well-balanced periods of cold and warmth.

The POM value for GH12 is more than $1^{\circ} \mathrm{C}$ higher than that inferred for the other boreholes independently on which SAT time series (Florence or Genoa) is adopted as a forcing function. This is probably due to a too high flow velocity (upward), which causes violation of the assumed upper boundary temperature conditions, rather than a really occurred climatic change. The estimated Darcy velocity is almost similar in both the SAT hypotheses. The largest differences were obtained for GH10 and GH12. This implies possible small variations of permeability with time which may either decrease or increase because of mineral precipitation or solutional phenomena (see e.g. Fetter, 1988, for a review).

POM calculations for borehole GH13 were based on the SAT time series from the nearby Bobbio observatory. The low correlation between the SAT records measured at Bobbio and Genoa and the position of the two stations, at the Ligurian coast and in the Apennines chain, respectively, imply large climatic differences and argue against the use of the longer Genoa SAT series as forcing function for GH13. POM was estimated both for the 1982 and 2002 borehole temperature log.

For a conductive/convective thermal regime, the estimated POM, representing the long-term surface temperature before 1934 , is $12.0^{\circ} \mathrm{C}$ for the 1982 thermal $\log$ and $12.6^{\circ} \mathrm{C}$ for the most recent record. The reference mean temperature (19702000) for the Bobbio series is $12.3^{\circ} \mathrm{C}$. This implies that the long-term temperatures before 1930s was from slightly lower to similar to the last 30-year average. The 1934-1950, 19501970 and 1970-1990 mean SATs at Bobbio were 11.5, 11.7 and $12.0^{\circ} \mathrm{C}$, respectively. Thus, significant cooling occurred between 1934 and 1950, and since 1970 the temperature achieved almost the same level of the period prior to 1934 .

Moreover, our calculations indicate only a slight upward flow for the borehole GH13, at a rate of 0.14$0.21 \times 10^{-9} \mathrm{~m} \mathrm{~s}^{-1}$. This conclusion is corroborated by the POM estimated with a purely conductive approach, which gives a long-term average of $12.0^{\circ} \mathrm{C}$ for both the 1982 and 2002 thermal logs. These POM values fall within the error bars or are very close to values calculated using the conductive/advective analysis. The observed reduced temperature are well matched by the temperature calculated both with the pure conductive and the conductive/advective approach (Fig. 3b), and the rms misfits are low (0.06 and $0.04 \mathrm{~K}$, respectively).

POM calculations for the boreholes GH14 and GH15 were made on basis of the 2002 thermal logs. The SAT records of
Table 3. The same as Table 2, with POM temperatures before 1900.

\begin{tabular}{llll}
\hline Borehole & $\operatorname{POM}_{c}\left({ }^{\circ} \mathrm{C}\right)$ & $\operatorname{POM}_{a}\left({ }^{\circ} \mathrm{C}\right)$ & $v\left(10^{-9} \mathrm{~m} \mathrm{~s}^{-1}\right)$ \\
\hline GH9 & 15.0 & 15.1 & -0.03 \\
GH10 & 15.1 & 14.9 & 0.12 \\
GH11 & 15.0 & 15.0 & 0.00 \\
GH12 & 16.4 & 16.8 & -0.89 \\
\hline
\end{tabular}

the meteorological observatory of Genoa located near these boreholes were used as a forcing function. The POM estimate for the borehole GH14 (Fig. 7) gives a very high value $\left(16.6^{\circ} \mathrm{C}\right)$ that implies a temperature prior to 1833 of $0.7 \mathrm{~K}$ higher to the average $\left(15.8^{\circ} \mathrm{C}\right)$ of the period $1970-2000$. Even if such a POM is well comparable to that inferred by Pasquale et al. (2000) from a nearby borehole, the inferred Darcy velocity is so high $\left(-2.02 \times 10^{-8} \mathrm{~m} \mathrm{~s}^{-1}\right)$ and the depth of the borehole so shallow that this result should be treated with caution.

Calculations for the borehole GH15 give a non-unique solution with rms minima corresponding to high Darcy velocity $\left(>10^{-9} \mathrm{~m} \mathrm{~s}^{-1}\right)$. This result agrees with the temperature fluctuations observed between the 2001 and 2002 thermal logs and points to the possibility of intensive water flow, which may render our approach inapplicable.

\subsection{GST histories}

Figure 8 shows GST histories obtained from inversion of the thermal logs of the holes GH9, GH11 and GH13, in which the effect of water movement is practically negligible. The results are compared with previous reconstructions obtained by Pasquale et al. (2005a) for neighbouring holes (GH1, GH3, and GH4) with another inversion technique.

The GST histories of GH9 and GH11 show a similar trend. Because of the relatively shallow depth of the measured temperature logs, climatic changes cannot be resolved before 1500 A.D. The boreholes recorded a warm period from 1700 to 1850 , followed by cooling from 1890 to 1970 . The temporary temperature increase in 1940s, clearly visible in the Florence SAT records, was probably too short to be resolved with inversion. Before the 18-19th century, temperatures were generally higher and similar to conditions occurred in the 1960-1990 reference period. This corroborates the results obtained with the POM technique. The more recent climatic changes shown by the GST history curves, i.e. a rapid warming detected in all boreholes since about 1950-1970, are coherent with the temperature increase observed in Florence.

Figure 8 also shows a comparison of the GST histories obtained from the logs recorded in 1982 and 2002 in the borehole GH13 with that inferred by Pasquale et al. (2005a) for the borehole GH1 which is about $50 \mathrm{~km}$ apart. High 

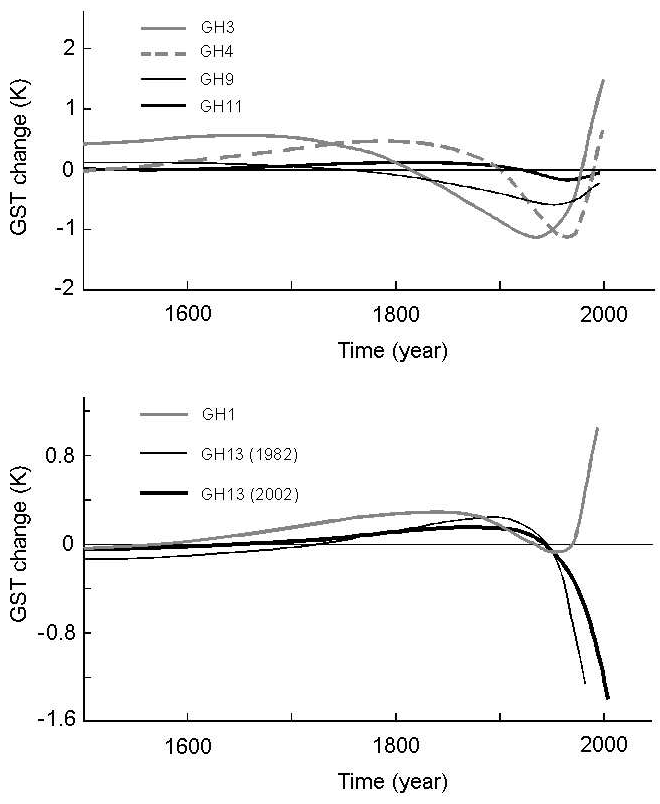

Fig. 8. GST histories for boreholes GH9, GH11 and GH13. Results for GH1, GH3 and GH4 from Pasquale et al. (2005a) are shown for comparison.

coincidence of GSTs reconstructed from "old" and "new" temperature logs significantly increases the confidence of the reconstructed climatic histories. The three GST history curves show a coherent climatic signal until 1950, namely slightly colder conditions before the $16-17$ th century, a temperature increase of $0.4-0.6 \mathrm{~K}$ until 1880 , followed by cooling. The warmer climate inferred for the period before the second half of the 20th century is in agreement with the POM results for the same borehole. After 1940-1950, the GST histories of GH13 and GH1 show an opposite trend. Cooling continues in the former borehole, whereas warming was inferred in the latter.

\section{Discussion and conclusions}

A significant number of available borehole temperature data has been so far considered of no use for studies on climatic changes, because they were suspected of being biased by groundwater flow. This reduced noticeably the number of suitable boreholes even in areas where extensive thermal logging was performed. Conversely, hydraulic disturbances in the temperature-depth profiles recorded in boreholes can be of help in groundwater studies for the estimation of flow parameters (Reiter, 2001; Anderson 2005; Verdoya et al., 2007).

Kohl (1998) made a first attempt to include also boreholes disturbed by water circulation in the climate studies by taking into account the combined effect of conductive heat transfer and advection. He demonstrated that, with the exception of some extreme cases, such as strong topography change or advection, the climatic signal cannot be totally masked by hydraulic flow and can be successfully recovered from the temperature logs. On the other hand, if thermal data are affected by advection, the paleoclimate reconstructions from a purely conductive approach may be seriously biased even in the case of a strong climatic signal (Lewis and Wang, 1992).

The advective/conductive approach permits to widen the geothermal dataset available for climatic studies as well as to recognise possible hydraulic disturbances from the analysis of precision temperature logs in areas with no information on groundwater movement. However, in hydrogeological systems, the intrinsic thermo-hydraulic properties of the medium and the ratio of the system depth to system length are factors that control the temperature distribution. The fluid velocity is also a function of depth, and vertical flow is satisfied only at recharge and discharge areas (Domenico and Palciauskas, 1973).

Bodri and Čermák (2005) evaluated the influence of 2-D fluid flow on the 1-D POM/velocity estimates for hydraulic gradients and Darcy velocities similar to those inferred for the boreholes investigated in this paper. They demonstrated that significant horizontal water flow might bias the POM estimates by only a few tenths of ${ }^{\circ} \mathrm{C}$. Even if the vertical component of the water velocity may vary with depth by a factor of two, the estimated velocities almost coincide or are slightly larger than the mean vertical velocities in the 0 $200 \mathrm{~m}$ range.

In substance, horizontal flow is not a first-order concern in the coupled POM and velocity calculations. In an almost flat, layered medium, the horizontal temperature gradients are considerably lower than the geothermal gradients. Accordingly, horizontal flow rates must be proportionally larger than vertical flow rates to have similar impact in the advective term of the heat transfer equation. Thus, values inferred from the analysis of the minimum rms misfit (see e.g. Fig. 7) correspond to real values for boreholes with principally vertical flow. For thermal logs significantly affected by horizontal water movement, the bias in the estimated POMs and velocities might be as large as $0.2-0.3 \mathrm{~K}$ and $0.4-0.5 \times 10^{-9} \mathrm{~m} \mathrm{~s}^{-1}$, respectively.

Besides hydraulic disturbances, the POM estimations can be also biased by other sources of errors. POM is the longterm mean surface temperature against which 19th and/or 20th century warming can be referenced. A basic assumption of this method is that the air temperature, used as a forcing function (surface boundary condition) track ground temperature. Thus, the POM value, calculated from the SAT and ground temperature records, reflects the climate change in the neighbourhood of a borehole. Non-climatic sources for decoupling of the air-ground temperatures were investigated in numerous works (e.g. Chisholm and Chapman, 1992; Harris and Chapman, 1998; Beltrami and Harris, 2001). In view of the insignificance of topographic corrections in our boreholes, air-ground temperature differences might arise, in 
principle, from temporary changes in the near surface conductive properties. However, as the examined boreholes were drilled in consolidated rocks, the subsurface layers cannot be affected by significant transient variations in moisture content and, correspondingly, in conductive properties. Effect of soil freezing is similarly insignificant in the investigated area (Pasquale et al., 2005a).

The analysis technique adopted in this paper provides further constraints to the climatic reconstruction in centralnorthern Italy using a set of temperature logs more or less affected by perturbations due to water circulation. The method used for the POM estimation is applicable for low-medium permeability and for relatively small hydraulic gradients, i.e. Darcy velocities of about $10^{-9} \mathrm{~m} \mathrm{~s}^{-1}$. These values are well below the velocity obtained for the borehole GH15, whose POM temperature may be thus by $1.0-1.5 \mathrm{~K}$ above its true value.

Our climatic reconstructions deduced with the POM technique argue against a clear evidence of the Little Ice Age in the central sector of Italy. This find a confirmation also in the GST curves obtained for boreholes GH9 and GH11. In the area under investigation, only the temperature decrease in the 19th century could be related to the last part of this period. The same result was also obtained with the analysis of a different set of boreholes by Bodri et al. (2003) and Pasquale et al. (2005a). The high coherence of the GST histories reconstructed for the Ligurian-Tyrrhenian side implies that this feature is typical for the whole investigated area and does not represent an artefact of the GST inversion.

The set of tree-rings reconstructions of the middle-ages temperature including the Marseille-Rome-Alps area revealed at least six episodes, two-three decade long, with oscillating climatic conditions from 1600 to 1800 A.D. (SerreBachet, 1994). The periods 1600-1630, 1650-1685 and 1710-1740 were characterized by warm to very warm climate. Because of the well-known decrease in resolution of the GST method into the past (e.g. Bodri and Čermák, 1995, and references therein), well balanced temperature variations can result in smoothed GST curves (Fig. 8).

On a shorter time scale, SAT oscillations may be not properly archived in the ground temperatures. This might occur for the results of inversion of the borehole GH13. The SAT time series of Bobbio shows cooling trend of $-0.14 \mathrm{~K} \mathrm{yr}^{-1}$ until 1950, and slight warming $\left(0.02 \mathrm{~K} \mathrm{yr}^{-1}\right)$ since then. This warming did not represent one-way story, but was accompanied by a number of provisional returns to colder conditions. This kind of cyclic signal can hardly be recovered from inversion (Pasquale et al., 2005a). The SAT series from Alessandria and Genoa also exhibit a cold episode between 1940 and 1960 with cooling rates comparable to Bobbio (i.e. $\left.-0.13 \mathrm{~K} \mathrm{yr}^{-1}\right)$. However, the subsequent warming at these sites has been rather constant $\left(0.02 \mathrm{~K} \mathrm{yr}^{-1}\right.$ in Genoa).

Moreover, the discrepancy in the GST histories and in the SAT series can be due to a variety of factors, such as variations of the agricultural activity or vegetation cover and urbanization in the surroundings of the borehole site. The change of vegetation and land use in the neighbouring of the investigated boreholes could produce significant temperature anomalies (e.g. Majorowicz and Skinner, 1997), which do not reflect regional air temperature variations. However, the quantitative description of the nature and the magnitude of such microclimatic effects is a difficult problem. All the boreholes analysed in this paper are located in rural areas, but except for the last twenty years, no detailed record is available about changes in land use. Thus, further studies are required before ascribing to local climatic effect the different GST history inferred for the borehole GH13.

In conclusion, the application of the conductive/advective analysis for POM estimations shows that method allows the possibility to include some of the previous rejected temperatures $\operatorname{logs}$ available for central-northern Italy for the paleoclimate analysis. This significantly increases the geothermal database suitable for the climate reconstruction and thus ensures more detailed knowledge of the past climate changes in the areas were independent climatic information is not available. The obtained results suggest that even advectively distorted borehole temperature log may still contain a valuable signal, which can be used in paleoclimate reconstruction to assess the POM-temperatures. Moreover, when independent hydrogeological information is not available, it can help to distinguish objectively boreholes characterised by a purely conductive thermal regime, whose temperature log can be subsequently inverted for the reconstruction of the GST history.

The results obtained for the set of boreholes scattered in the Ligurian-Tyrrhenian side of the Apennines chain indicate that the 19th century does not contain definite trends, with periods of cold and warmth well balanced in the long time scale. A similar result was obtained for borehole GH1 (Pasquale et al., 2005a). The "quiet" 19th century (at least after 1814) is probably characteristic for the whole LigurianTyrrhenian side. Our paleoclimatic reconstructions indicated warmer conditions preceding the period of meteorological observations in the area and comparable with the reference period of 1960-1990, cooling that began since 1850 and continued up to 1950, and warming since then. This general climatic course appears to be common for the whole centralnorthern Italy. Generally, cold climate is characteristic only for the mid 19th-mid 20th centuries. The apparent absence of Little Ice Age can be attributed to high variability in the area under investigation. This variability results also from independent climatic proxies such as tree-ring reconstructions. It is also appears that the recent climatic episodes may have partly a local origin and can obscure the changes forced by the regional atmospheric circulation.

Acknowledgements. We thank L. Bodri for her fundamental contribution to an earlier version of the manuscript and S. Bellani for making available original temperature logs.

Edited by: V. Rath 


\section{References}

Anderson, M. P.: Heat as a ground water tracer, Ground Water, 43(6), 951-968, 2005.

Baldi, P., Bellani, S., Ceccarelli, A., Fiordalisi, A., Squarci, P., and Taffi, L.: Nuovi dati geotermici nelle aree a Sud-Est ed a Sud del campo geotermico di Travale (Toscana), Atti del $13^{\circ}$ Convegno GNGTS, CNR, Roma, pp. 211-221, 1994.

Beltrami, H. and Harris, R. H. (Eds.): Inference of climate change from geothermal data (Special Issue), Global Planet. Change, 29, 149-348, 2001.

Bodri, L., Chiozzi, P., Pasquale, V., and Verdoya, M.: Last 1000 years climate reconstruction inferred from geothermal measurements in Italy, Proceedings of the 2nd Int. Conf. European Soc. for Environmental History, Prague 2003, pp. 16-22, 2003.

Bodri, L. and Čermák, V.: Climate changes in the last millennium inferred from borehole temperatures: results from the Czech Republic - part I, Global Planet. Change, 11, 111-125, 1995.

Bodri, L. and Čermák, V.: High frequency variability in recent climate and the north Atlantic oscillation, Theor. Appl. Climatol., 74, 33-40, 2003.

Bodri, L. and Čermák, V.: Borehole temperatures, climate change and pre-observational surface air temperature mean: allowance for hydraulic conditions, Global Planet. Change, 45, 265-276, 2005.

Bodri, L. and Dövényi, P.: Climate change of the last 2000 years inferred from borehole temperatures: data from Hungary, Global Planet. Change, 41, 121-133, 2004.

Bredehoeft, J. D. and Papadopulos, I. S.: Rates of vertical groundwater movement estimated from the earth's thermal profile, Water Resour. Res., 1, 325-328, 1965.

Carslaw, H. S. and Jaeger, J. C.: Conduction of heat in solids, Oxford Univ. Press New York, pp. 386, 1959.

Chisholm, T. J. and Chapman, D. S.: Climate change inferred from analysis of borehole temperatures: an example from western Utah. J. Geophys. Res., 97, 14 155-14 175, 1992.

Domenico, P. A. and Palciauskas, V. V.: Theoretical analysis of forced convective heat transfer in regional ground-water flow, Geol. Soc. Amer. Bull., 84, 3803-3814, 1973.

Ferguson, G., Woodbery, A. D., and Matile, G. L. D.: Estimating deep recharge rates beneath an interlobate moraine using temperature logs, Ground Water, 41, 640-646, 2003.

Fetter, C. W.: Applied Hydrology, 2nd Edition, Macmillan New York, pp. 592, 1988

Hansen, J. and Lebedeff, S.: Global trends of measured surface air temperature, J. Geophys. Res., 92, 13 345-13 372, 1987.

Harris, R. N. and Chapman, D. S.: Geothermics and climate change: Part 2. Joint analysis of borehole temperature and meteorological data, J. Geophys. Res., 103, 7371-7383, 1998.
Karl, T. R., Knight, R. W., and Plummer, N.: Trends in high frequency climate variability in the twentieth century, Nature, 377, 217-220, 1995.

Kohl, T.: Paleoclimatic temperature signals - can they be washed out?, Tectonophysics, 291, 225-234, 1998.

Kukkonen, I. T. and Joeleth, A.: Weichselian temperatures from geothermal heat flow data, J. Geophys. Res., 108(B3), 2163 , doi:10.1029/2001JB001579, 2003.

Lewis, T. J. and Wang, K.: Influence of terrain on bedrock temperatures, Global Planet. Change, 98, 87-100, 1992.

Menke, W.: Geophysical Data Analysis: Discrete Inverse Theory, Int. Geophys. Ser., 45, Academic Press, San Diego, pp. 289 , 1989.

Majorowicz, J. A. and Safanda, J.: Measured versus simulated transients of temperature logs - a test borehole climatology, J. Geophys. Eng., 2, 1-8, 2005.

Majorowicz, J. A. and Skinner, W. R.: Potential causes of the differences between ground and surface air temperature warming across different ecozones in Alberta, Canada, Glob. Planet. Change, 115, 79-91, 1997.

Moberg, A., Jones, P. D., Barriendos, M., Bergström, H., Camuffo, D., Cocheo, C., Davies, T. D., Demarée, G., Martin-Vide, J., Maugeri, M., Rodriguez, R., and Verhoeve, T.: Day-to-day temperature variability trends in 160- to 275-year-long European instrumental records, J. Geophys. Res., 105, 22 849-22 868, 2000.

Pasquale, V., Verdoya, M., and Chiozzi, P.: Climate change from meteorological observations and underground temperatures in Northern Italy, Stud. Geophys. Geod., 42, 30-40, 1998.

Pasquale, V., Verdoya, M., Chiozzi, P., and Safanda, J.: Evidence of climate warming from underground temperatures in NW Italy, Global Planet. Change, 25, 215-222, 2000.

Pasquale, V., Verdoya, M., Chiozzi, P., Bodri, L., and Bellani, S.: Temperature signal in the underground for climate history reconstruction in Italy, Global Planet. Change, 47, 36-50, 2005a.

Pasquale, V., Verdoya, M., and Chiozzi, P.: Orographic influence on the central-northern Italy climate from underground temperatures, Croatian Meteorological Journal, 30, 669-671, 2005b.

Reiter, M.: Using precision temperature logs to estimate horizontal and vertical groundwater flow components, Water Resour. Res., 37, 663-674, 2001

Reiter, M.: Possible ambiguities in subsurface temperature logs consideration of groundwater flow and groundsurface temperature change, Pure Appl. Geophys., 162, 343-355, 2005.

Serre-Bachet, F.: Middle ages temperature reconstructions in Europe, a Focus on Northeastern Italy, Clim. Change, 26, 213-224, 1994.

Verdoya, M., Chiozzi, P., and, Pasquale, V.: Inferring hydrogeothermal parameters from advectively perturbed thermal logs, Int. J. Earth Sci., in press, 2007. 